\title{
Magnetism and superconductivity of strongly correlated electrons on the triangular lattice
}

\author{
Cédric Weber, ${ }^{1}$ Andreas Läuchli, ${ }^{1}$ Frédéric Mila, ${ }^{2}$ and Thierry Giamarchi ${ }^{3}$ \\ ${ }^{1}$ Institut Romand de Recherche Numérique en Physique des Matériaux (IRRMA), CH-1015 Lausanne, Switzerland \\ ${ }^{2}$ Institute of Theoretical Physics, EPFL, CH-1015 Lausanne, Switzerland \\ ${ }^{3}$ DPMC, University of Geneva, Quai Ernest Ansermet 24, CH-1211 Geneva, Switzerland \\ (Received 28 September 2005; published 20 January 2006)
}

\begin{abstract}
We investigate the phase diagram of the $t$ - $J$ model on a triangular lattice using a variational Monte Carlo approach. We use an extended set of Gutzwiller projected fermionic trial wave functions allowing for simultaneous magnetic and superconducting order parameters. We obtain energies at zero doping for the spin-1/2 Heisenberg model in very good agreement with the best estimates. Upon electron doping (with a hopping integral $t<0$ ) this phase is surprisingly stable variationally up to $n \approx 1.4$, while the $d_{x^{2}-y^{2}}+i d_{x y}$ order parameter is rather weak and disappears at $n \approx 1.1$. For hole doping, however, the coplanar magnetic state is almost immediately destroyed and $d_{x^{2}-y^{2}}+i d_{x y}$ superconductivity survives down to $n \approx 0.8$. For lower $n$, between 0.2 and 0.8 , we find saturated ferromagnetism. Moreover, there is evidence for a narrow spin density wave phase around $n \approx 0.8$. Commensurate flux phases were also considered, but these turned out not to be competitive at finite doping.
\end{abstract}

DOI: 10.1103/PhysRevB.73.014519

PACS number(s): 74.72.-h, 71.10.Fd, 74.25.Dw

\section{INTRODUCTION}

The discovery of high- $T_{c}$ superconductivity, and the observation ${ }^{1}$ that strong correlations are important in connection with these compounds has led to a tremendous interest in understanding strongly correlated electron physics. In particular the two simplest models for strongly correlated electrons, namely, the Hubbard and $t-J$ models, have been the subject of intensive studies.

For example, one question of crucial interest is the interplay between superconductivity and antiferromagnetism close to the insulating phase in the $t-J$ model. The ground state of this model on the square lattice is known to be antiferromagnetic at half filling and one of the important questions is what happens upon doping. All the approaches to these strong coupling problems involve approximations, and it is sometimes difficult to distinguish the artifact due to approximations from the true features of the model. However, for the case of the square lattice, both the variational Monte Carlo method ${ }^{2,3}$ (VMC) and mean-field theories ${ }^{4}$ have found a $d$-wave superconducting phase in the $t-J$ model. A wave function combining antiferromagnetism and superconductivity was proposed for the Hubbard and $t-J$ models, ${ }^{5,6}$ allowing us to reconcile the variational results between these two models.

This wave function allowed for an excellent variational energy and order parameter. Moreover, a range of coexistence between superconductivity and antiferromagnetism was found: the antiferromagnetism disappears at $8 \%$ doping and the RVB state survives up to $40 \%$ doping.

Although no definite answer can be given on that point, since no exact solution is available away from half filling, the coexistence region is indeed most likely an artifact of the restricted variational space. It would be probably replaced by a pure antiferromagnetic phase if additional elements (incommensurability of the antiferromagnetism, inhomogeneous $\mathrm{AF}$ order parameter, etc.) were incorporated in the wave function. Note that the coexistence feature is removed ${ }^{7}$ when small next-nearest-neighbor and third-neighbor hopping terms are added to the $t$ - $J$ model, using the same class of variational wave functions.

Further investigations of this class of wavefunctions has been very fruitful for the square lattice. This allowed us to successfully compare to some of the experimental features with the high- $T_{c}$ cuprates, ${ }^{8,9}$ even if of course many questions remain regarding the nature of the true ground state of the system.

The resonating valence bond (RVB) scenario proposed by Anderson ${ }^{1}$ was argued to be even more relevant in the geometry of the triangular lattice. At half filling, the lattice is a frustrated magnet: the competition between the exchange integrals leads to unsatisfied bonds. The original expectation is that quantum fluctuations might lead to a spin-liquid behavior. However, at half filling, it appears by now that the spin$1 / 2$ triangular lattice has a three sublattice coplanar magnetic order. ${ }^{10-12}$ Quantum fluctuations are nevertheless strong, and the sublattice magnetization is strongly reduced due to these fluctuations. It is therefore expected that the magnetism is fragile and quickly destroyed by doping and that a strong RVB instability is present. Indeed, RVB mean field theories ${ }^{13-15}$ were used for the $t-J$ model, and $d_{x^{2}-y^{2}}+i d_{x y}$ pairing was found over a significant range of doping. The same approach and questions that arise in the framework of the $t-J$ model on the square lattice are thus very relevant in the present frustrated lattice. The success of the variational approach for the square lattice suggests to investigate the same class of variational wave functions for the triangular one.

In addition to its own theoretical interest, another motivation for understanding the physics of electrons on a triangular lattice is provided by the recent discovery of superconductivity at low temperature in the $\mathrm{CoO}_{2}$ layered compounds ${ }^{16}\left(\mathrm{Na}_{\delta} \mathrm{CoO}_{2} \cdot y \mathrm{H}_{2} \mathrm{O}\right)$. In these systems, superconductivity is observed in a range of electron doping $\delta$ between 25 and $33 \% .{ }^{17} \mathrm{Na}_{\delta} \mathrm{CoO}_{2} \cdot y \mathrm{H}_{2} \mathrm{O}$ consists of two-dimensional 
(2D) $\mathrm{CoO}_{2}$ layers separated by thick insulating layers of $\mathrm{Na}^{+}$ ions and $\mathrm{H}_{2} \mathrm{O}$ molecules. It is a triangular net of edge sharing oxygen octahedra; Co ions are at the center of the octahedra forming a $2 \mathrm{D}$ triangular lattice. Takada et al. ${ }^{16}$ speculated that this system might be viewed as a doped spin-1/2 Mott insulator. Based on LDA calculations, ${ }^{18}$ a simplified single band $t-J$ picture with negative $t$ and electron doping was put forward. ${ }^{13-15}$ Such systems might thus be the long-sought low-temperature resonating valence bond superconductor, on a lattice which was at the basis of Anderson's original ideas on a possible quantum spin liquid state. ${ }^{19,20}$

We propose in this paper to study the $t$ - $J$ model within the framework of the variational Monte Carlo (VMC) method, which provides a variational upper bounds for the groundstate energy. In contrast to mean-field theory, it has the advantage of exactly treating the no double-occupancy constraint. VMC using simple RVB wave functions has been used for the triangular lattice ${ }^{21}$ and it was found that $d_{x^{2}-y^{2}}$ $+i d_{x y}$ superconductivity is stable over a large range of doping. However, in the previous study the fact that the $t-J$ is magnetically ordered at half filling was not taken into account. We expect that the frustration in the triangular lattice may lead to a richer phase diagram and to many different instabilities. We thus propose here to study extended wave functions containing at the same time magnetism, flux phase and RVB instabilities, in a similar spirit as for the square lattice, 5,6 in order to study in detail the interplay between frustrated magnetism and superconductivity. Given the noncollinear nature of the magnetic order parameter compared to the case of the square lattice, the task is, however, much more complicated. We thus present in this work a general mean-field Hamiltonian which takes into account the interplay between magnetic, RVB, and flux-phase instabilities. The resulting variational wave function is sampled with an extended VMC, which uses Pfaffian updates rather than the usual determinant updates. We show that the interplay between the different instabilities leads to a faithful representation of the ground state at half filling, and we also find good variational energies upon doping. To benchmark our wave function, we carry out exact diagonalizations on a small 12 sites cluster and compare the variational energies and the exact ones. Finally, a commensurate spin density wave is considered, and is shown to be relevant for the case of hole doping.

The outline of the paper is as follows. In Sec. II we present the model and the numerical technique. In Sec. III we show the variational results both for the case of hole and electron doping. Finally Sec. IV is devoted to the summary and conclusions.

\section{MODEL AND METHOD}

We study the $t-J$ model on the triangular lattice defined by the Hamiltonian

$$
H_{t-J}=-t \sum_{\langle i, j\rangle, \sigma}\left(c_{i, \sigma}^{\dagger} c_{j, \sigma}+\text { H.c. }\right)+J \sum_{\langle i, j\rangle}\left(\mathbf{S}_{i} \cdot \mathbf{S}_{j}-\frac{1}{4} n_{i} n_{j}\right) \text {. }
$$

The model describes electrons hopping with an amplitude $t$, and interacting with an antiferromagnetic exchange term $J$

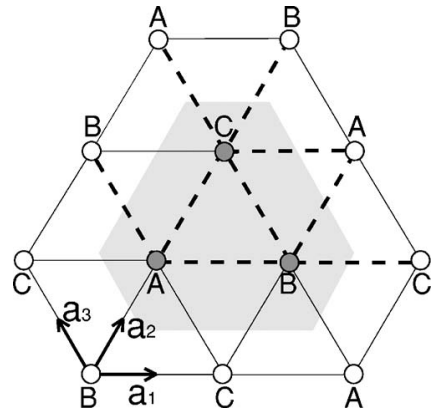

FIG. 1. Three-site supercell of the triangular lattice. The onsite magnetic variational parameters can vary independently on each of the site $A, B$, and $C$ of the supercell. The BCS pairing as well as the flux vary independently on each of the different dashed bonds.

between nearest-neighbor sites (denoted $\langle i, j\rangle$ ) of a triangular lattice. $\mathbf{S}_{i}$ denotes the spin at site $i, \mathbf{S}_{i}=1 / 2 c_{i, \alpha}^{\dagger} \vec{\sigma}_{\alpha, \beta} c_{i, \beta}$, and $\vec{\sigma}$ is the vector of Pauli matrices. $H_{t-J}$ is restricted to the subspace where there are no doubly occupied sites. In order to simplify the connection to the Cobaltates we set $t=-1$ in the following and present the results as a function of the electron density $n \in[0,2]$, half filling corresponding to $n=1 . n>1$ corresponds to a $t-J$ model at $\tilde{n}=2-n$ for $t=1$, by virtue of a particle-hole transformation. In the first part of this section, we emphasize on the method to construct a variational wave function containing both superconductivity and noncollinear magnetism. The wave function allows us to consider three-sublattice magnetism, however, since the latter wave function is restricted to a three site supercell, we briefly comment on a second simpler variational wave function type, which allows us to describe commensurate spin order. In the second part of the section, we define the relevant instabilities and the corresponding order parameters.

\section{A. Variational wave function}

In order to study this model we use a variational wavefunction built out of the ground state of the following meanfield-like Hamiltonian:

$$
\begin{aligned}
H_{\mathrm{MF}}= & \sum_{\langle i, j\rangle, \sigma}\left(-t e^{\left.i \theta_{i, j}^{\sigma} c_{i \sigma}^{\dagger} c_{j \sigma}+\text { H.c. }\right)}\right. \\
& +\sum_{\langle i, j\rangle, \sigma, \sigma^{\prime}}\left(\left\{\Delta_{\sigma, \sigma^{\prime}}\right\}_{i, j} c_{i \sigma}^{\dagger} c_{j \sigma^{\prime}}^{\dagger}+\text { H.c. }\right) \\
& +\sum_{i} \mathbf{h}_{i} \cdot \mathbf{S}_{i}-\mu \sum_{i, \sigma} n_{i, \sigma} .
\end{aligned}
$$

$H_{\mathrm{MF}}$ contains at the same time BCS pairing $\left(\boldsymbol{\Delta}_{i, j}=\left\{\Delta_{\sigma, \sigma^{\prime}}\right\}_{i, j}\right)$, an arbitrary external magnetic field $\left(\mathbf{h}_{i}\right)$, and arbitrary hopping phases $\left(\theta_{i, j}^{\sigma}\right)$, possibly spin dependent. These variational parameters are unrestricted on the $A, B, C$ sites and the corresponding bonds of a three-site supercell, as shown in Fig. 1. We allow both singlet $\left(\Delta_{i, j}^{(S=0)}\right)$ and general triplet $\left(\boldsymbol{\Delta}_{i, j}^{(S=1)}\right)$ pairing symmetries to be present. They correspond to choosing 


$$
\begin{gathered}
\boldsymbol{\Delta}_{i, j}^{(S=0)}=\left(\begin{array}{cc}
0 & \psi_{i, j} \\
-\psi_{i, j} & 0
\end{array}\right), \\
\boldsymbol{\Delta}_{i, j}^{(S=1)}=\left(\begin{array}{cc}
\psi_{i, j}^{2} & \psi_{i, j}^{1} \\
\psi_{i, j}^{1} & \psi_{i, j}^{3}
\end{array}\right) .
\end{gathered}
$$

Since $H_{\mathrm{MF}}$ is quadratic in fermion operators it can be solved by a Bogoliubov transformation. In the most general case considered here, this gives rise to a $12 \times 12$ eigenvalue problem, which we solve numerically. We then find the ground state of $H_{\mathrm{MF}}$

$$
\left|\psi_{\mathrm{MF}}\right\rangle=\exp \left\{\sum_{i, j, \sigma_{i}, \sigma_{j}} a_{\left(i, j, \sigma_{i}, \sigma_{j}\right)} c_{i \sigma_{i}}^{\dagger} c_{j \sigma_{j}}^{\dagger}\right\}|0\rangle .
$$

Here $a_{\left(i, j, \sigma_{i}, \sigma_{j}\right)}$ are numerical coefficients. Note that $\left|\psi_{\mathrm{MF}}\right\rangle$ has neither a fixed number of particles due to the presence of pairing, nor a fixed total $S^{z}$ due to the noncollinear magnetic order. Thus in order to use it for the VMC study we apply to it the following projectors: $\mathcal{P}_{N}$ which projects the wave function on a state with fixed number of electrons and $\mathcal{P}_{S^{z}}$ which projects the wave function on the sector with total $S^{z}=0$. Finally we discard all configurations with doubly occupied sites by applying the complete Gutzwiller projector $\mathcal{P}_{\mathcal{G}}$. The wave function we use as an input to our variational study is thus

$$
\begin{aligned}
\left|\psi_{\text {var }}\right\rangle & =\mathcal{P}_{\mathcal{G}} \mathcal{P}_{S^{z}} \mathcal{P}_{N}\left|\psi_{\mathrm{MF}}\right\rangle \\
& =\mathcal{P}_{\mathcal{G}} \mathcal{P}_{S^{z}}\left\{\sum_{i, j, \sigma_{i}, \sigma_{j}} a_{\left(i, j, \sigma_{i}, \sigma_{j}\right.} c_{i \sigma_{i}}^{\dagger} c_{j \sigma_{j}}^{\dagger}\right\}^{N / 2}|0\rangle .
\end{aligned}
$$

Although the wave function (5) looks formidable, it can be reduced to a form suitable for VMC calculations. Using

$$
\langle\alpha|=\langle 0| c_{k_{1}, \sigma_{1}} \cdots c_{k_{N}, \sigma_{N}},
$$

we find that

$$
\begin{gathered}
\left\langle\alpha \mid \psi_{\mathrm{var}}\right\rangle=P_{f}(\mathbf{Q}) \\
Q_{i, j}=a_{\left(k_{i}, k_{j}, \sigma_{i}, \sigma_{j}\right)}-a_{\left(k_{j}, k_{i}, \sigma_{j}, \sigma_{i}\right)},
\end{gathered}
$$

where $P_{f}(\mathbf{Q})$ denotes the Pfaffian of the matrix $\mathbf{Q}$. Using this last relation, the function (5) can now be evaluated numerically using a Monte Carlo procedure with Pfaffian updates, as introduced in Ref. 22. In the particular case where $a_{k, l, \uparrow, \uparrow}=a_{k, l, \downarrow, \downarrow}=0$ and at $S^{z}=0$ (this happens if the BCS pairing is of singlet type and the magnetic order is collinear), the Pfaffian reduces to a simple determinant, and the methods becomes equivalent to the standard variational Monte Carlo $^{23}$ technique.

The above mean field Hamiltonian and wave function contain the main physical ingredients and broken symmetries we want to implement in the wavefunction. In order to further improve the energy and allow for out of plane fluctuations of the magnetic order we also add a nearest-neighbor spin-dependent Jastrow ${ }^{24}$ term to the wave function:

$$
\mathcal{P}_{\mathcal{J}}=\exp \left(\alpha \sum_{\langle i, j\rangle} S_{i}^{z} S_{j}^{z}\right)
$$

where $\alpha$ is an additional variational parameter. Our final wave function is thus

$$
\left|\psi_{\text {var }}\right\rangle=\mathcal{P}_{\mathcal{J}} \mathcal{P}_{S^{z}} \mathcal{P}_{N} \mathcal{P}_{\mathcal{G}}\left|\psi_{\mathrm{MF}}\right\rangle
$$

When $\alpha<0$ the Jastrow factor favors all configurations which belong to the ground-state manifold of a classical Ising antiferromagnet on the triangular lattice. Such a manifold is exponentially large ${ }^{25}$ and this Jastrow factor thus provides a complementary source of spin fluctuations.

In what follows we use the wave function (9) directly for the VMC, but we also examine improved wave functions with respect to Eq. (9) that can be obtained by applying one or more Lanczos steps: ${ }^{26-28}$

$$
|1 L s\rangle=\left(1+\lambda H_{t-J}\right)\left|\psi_{\mathrm{var}}\right\rangle
$$

with optimized ${ }^{29} \lambda$. Since the calculation of Lanczos step wave functions beyond the first step is very time consuming, most of the results we will present here were obtained using a single Lanczos step.

In the following, to clearly indicate which wave function we use, we will denote them in the following way: $\mathrm{MF} / J / n \mathrm{Ls}$, where MF denotes the fields present in the meanfield-like Hamiltonian $H_{\mathrm{MF}}, J$ is present if we use the Jastrow factor, $n$ Ls denotes the presence and the number of Lanczos steps applied on top of the bare wave function.

As usual with the VMC procedure, these general wave functions are now used to minimize the expectation value of the total energy $\left\langle H_{t-J}\right\rangle$ by changing the variational parameters. We used a correlated measurement technique ${ }^{5,6,30}$ combined with parallel processing to smoothen the energy landscape and use a steepest-descent-type routine to locate the minimum of energy. We then define the condensation energy $e_{c}$ of the optimal wave function as

$$
e_{c}=e_{\mathrm{var}}-e_{\mathrm{Gutzwiller}},
$$

where $e_{\text {Gutzwiller }}$ is the energy of the Gutzwiller wave function, i.e., the fully projected Fermi sea at zero magnetization. In some cases we had to keep a small BCS pairing field to avoid numerical instabilities. Let us note that the linear size of the $\mathbf{Q}$ matrix is two times larger than in the simpler case of determinantal update VMC. Therefore our largest 108 sites cluster with Pfaffian updates corresponds roughly to a 200 sites cluster using standard updates.

\section{B. Commensurate order}

Since the mean-field Hamiltonian (2) is restricted to a three site supercell, we investigated also a second class of mean-field Hamiltonians based on collinear commensurate structures, which are not contained in the previous Hamiltonian. For this type of phase, we used a simpler mean-field ansatz along the lines of Ref. 6. The mean-field Hamiltonian written in $k$ space is 


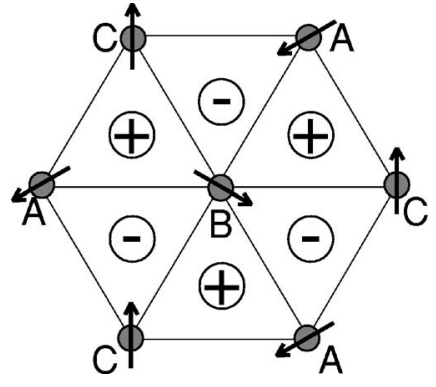

FIG. 2. The variational parameters $\mathbf{h}_{i}$ for the coplanar $120^{\circ}$ antiferromagnetic order. The spins lie in the $x-y$ plane. The $z$ component of the vector chirality $( \pm 1)$ on each triangular plaquette forms a staggered pattern.

$$
\begin{aligned}
H_{\mathrm{SDW}}= & \sum_{k, \sigma}\left[\left(\epsilon_{k}-\mu\right) c_{k \sigma}^{\dagger} c_{k \sigma}+f(Q, \sigma) c_{k+Q \sigma}^{\dagger} c_{k \sigma}\right] \\
& +\sum_{k}\left(\Delta_{k} c_{k \uparrow}^{\dagger} c_{-k \downarrow}^{\dagger}+\text { H. c. }\right),
\end{aligned}
$$

where $k$ does run over the Brillouin zone of the original triangular lattice, $\epsilon_{k}$ is the dispersion of the free electron Hamiltonian, and $\Delta_{k}$ is the Fourier transform of $\Delta_{i, j}$. Depending on $f(Q, \sigma)$, the ground state of the Hamiltonian is a commensurate charge density wave $[f(Q, \sigma)=f(Q)]$ or a spin density wave $[f(Q, \sigma)=\sigma f(Q)]$.

At half filling, we considered also several commensurate flux phases with $2 \pi \times q / p$ flux per plaquette, using the Landau gauge ${ }^{37}$ with $p \in\{2, \ldots, 10\}$ and $q<p$. The one with the lowest energy was found to be the $q=1, p=4$, as predicted theoretically, ${ }^{31}$ giving an energy close to the simple $d_{x^{2}-y^{2}}$ $+i d_{x y}$ wave function. Upon doping, however, the energy of such commensurate flux phases are rapidly much worse than the energies of our best wave functions. The main reason for this poor performance upon doping is the rather bad kinetic energy of these wave functions.

\section{Characterization of the encountered instabilities}

By minimizing all the variational parameters of the meanfield Hamiltonians (2) and (12) on a 12 and a 48 site lattice, we find that the relevant instabilities present at the mean field level consist of the following. (i) A $120^{\circ}$ coplanar antiferromagnetic order (AF), represented in Fig. 2. (ii) A concomitant staggered spin flux phase instability (SFL) with $\theta_{i, i+a_{1}, \sigma}=\theta \sigma, \theta_{i, i+a_{2}, \sigma}=-\theta \sigma, \theta_{i, i+a_{3}, \sigma}=\theta \sigma$. These bond phase factors correspond to a spin current in the $z$ direction which is staggered on elementary triangles of the triangular lattice. This instability follows rather naturally, since the $120^{\circ}$ antiferromagnetic state itself already displays the same staggered spin currents $\left(\mathbf{S}_{i} \times \mathbf{S}_{j}\right)^{z}$ on the nearest neighbor bonds (see Fig. 2). The effect of this instability was rather small and visible only at half filling. (iii) A translationally invariant superconducting phase with $d_{x^{2}-y^{2}}+i d_{x y}$ singlet pairing symmetry $\left(d^{+}\right)$, as well as the $d_{x^{2}-y^{2}}-i d_{x y}\left(d^{-}\right)$. We have also looked extensively for triplet pairing for both electron and hole dopings and low $J /|t| \leqslant 0.4$ on a 48 site cluster, but with no success. The minimum energy was always found for singlet pairing symmetry. (iv) A ferromagnetic state with partial or full polarization $(F)$. (v) A commensurate collinear spin density wave $^{32}$ (SDW) instability with wave vector $\mathbf{Q}_{N}=(\pi,-\pi / \sqrt{3})$.

\section{Order parameters}

In order to characterize the phases described by the optimal wave functions after projection, we have calculated the following observables.

The sublattice magnetization of the $120^{\circ}$ coplanar antiferromagnetic order

$$
M_{\mathrm{AF}}=\frac{1}{N} \sum_{i}\left\|\frac{\left\langle\psi_{\mathrm{var}}\left|\mathbf{S}_{i}\right| \psi_{\mathrm{var}}\right\rangle}{\left\langle\psi_{\mathrm{var}} \mid \psi_{\mathrm{var}}\right\rangle}\right\| .
$$

We have checked that the projected magnetization has the correct $120^{\circ}$ symmetry. To simplify the calculations, this expectation value has been sampled using wave functions without the projector $\mathcal{P}_{S z}$. We have checked that this gives the same result as the correlation function $\lim _{r \rightarrow \infty} \sqrt{\mathbf{S}_{i} \cdot \mathbf{S}_{i+r}}$ calculated with the projector $\mathcal{P}_{S z}$.

The $z$ component of the vector chirality (spin twist) on nearest-neighbor bonds

$$
\chi=\frac{1}{3 N} \sum_{i, \alpha}\left|\frac{\left.\left\langle\psi_{\mathrm{var}}\right| \mathbf{S}_{i} \times \mathbf{S}_{i+a_{\alpha}}\right)^{z}\left|\psi_{\mathrm{var}}\right\rangle}{\left\langle\psi_{\mathrm{var}} \mid \psi_{\mathrm{var}}\right\rangle}\right| .
$$

We have checked that the measured vector chirality has the symmetry of the staggered currents derived from the $120^{\circ}$ coplanar structure (see Fig. 2).

The amplitude of the absolute value of the collinear magnetization in the spin density wave wave function

$$
M_{\mathrm{SDW}}=\frac{1}{N} \sum_{i}\left|\frac{\left\langle\psi_{\mathrm{var}}\left|S_{i}^{z}\right| \psi_{\mathrm{var}}\right\rangle}{\left\langle\psi_{\mathrm{var}} \mid \psi_{\mathrm{var}}\right\rangle}\right| .
$$

The amplitude of the singlet superconducting order parameter

$$
\Delta=\sqrt{\frac{1}{4 N}\left|\lim _{r \rightarrow \infty} \sum_{i} \frac{\left\langle\psi_{\mathrm{var}}\left|\Delta_{i, \alpha}^{\dagger} \Delta_{i+r, \beta}\right| \psi_{\mathrm{var}}\right\rangle}{\left\langle\psi_{\mathrm{var}} \mid \psi_{\mathrm{var}}\right\rangle}\right|},
$$

where

$$
\Delta_{i, \alpha}^{\dagger}=c_{i, \uparrow}^{\dagger} \uparrow_{i+a_{\alpha} \downarrow}^{\dagger}-c_{i, \downarrow}^{\dagger} c_{i+a_{\alpha},}^{\dagger} \uparrow .
$$

The angular dependence of the real space correlations corresponds to those of the unprojected pairing symmetry. We have also checked that the value of $\Delta$ is independent of the choice of $\alpha$ and $\beta$.

\section{RESULTS AND DISCUSSION}

\section{A. Half filling}

We consider in this section the Heisenberg model (which is the limit of the $t-J$ model at half filling, up to a constant). The comparison with the large body of existing results for the Heisenberg model allows us to benchmark the quality of our wave function. 


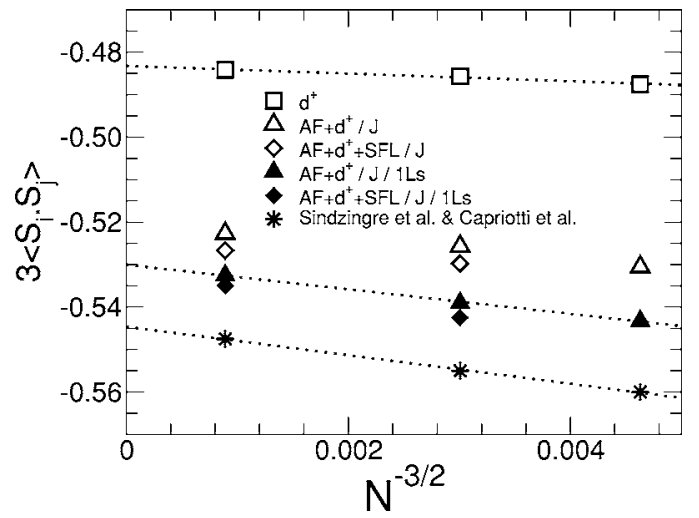

FIG. 3. Energy per site $e=3\left\langle\mathbf{S}_{i} \cdot \mathbf{S}_{j}\right\rangle$ of the different variational wave functions for the Heisenberg model versus the system size $N^{-3 / 2}$, with $N=36,48,108$ sites. Ordered by increasing condensation energy we find $d^{+}$(open squares), $\mathrm{AF}+d^{+} / J$ (open triangles), $\mathrm{AF}+d^{+}+\mathrm{SFL} / J$ (open diamonds), $\mathrm{AF}+d^{+} / J / \mathrm{Ls}$ (full triangles) and the $\mathrm{AF}+d^{+}+\mathrm{SFL} / J / \mathrm{Ls}$ (full diamonds). The stars are the best estimates of the ground-state energy available in the literature (Refs. 12 and 33).

Let us first briefly discuss the symmetry of the variational parameters at half filling. The variational magnetic field minimizes the energy for the two degenerate $120^{\circ}$ configurations. We found that the BCS pairing symmetry in the presence of AF order is of $d^{+}$type, whereas the $d^{-}, d_{x^{2}-y^{2}}$, and $d_{x y}$ pairings have close but higher energies. Finally, a staggered spin flux variational order improves a little bit the energy. Interestingly, this latter variational order is present in the ground state of the classical Heisenberg model. However, this instability was only relevant at half filling, and the energy gain for finite doping $(\delta>0)$ is not significant. The various energies for these wavefunctions are shown in Fig. 3. The $\mathrm{AF}+d^{+}+\mathrm{SFL} / J / \mathrm{Ls}$ wave function is thus the best approximation, within our variational space, of the ground state of the Heisenberg model. We compare its energy with other estimates of the ground-state energy in the literature (see Fig. 3 and Table I). The mixture of AF and $d^{+}$instabilities is improving a lot the energy, and our wave function has

TABLE I. Comparison of the average energy $3\left\langle S_{i} \cdot S_{j}\right\rangle$ and the average magnetization $M_{\mathrm{AF}}$ for the Heisenberg model ( $t-J$ model at half filling) in different recent works for the 36 site cluster and the extrapolation to the thermodynamic limit. The energy and the sublattice magnetization are measured for our best wave function (WF) $\left(\mathrm{AF}+d^{+}+\mathrm{SFL} / J / 1 L s\right)$ at half filling.

\begin{tabular}{lcc}
\hline \hline & $\left\langle 3 S_{i} \cdot S_{j}\right\rangle$ & $M_{\mathrm{AF}}$ \\
\hline 36 sites lattice & & \\
our best WF & $-0.543(1)$ & 0.38 \\
Capriotti et al. (Ref. 12) & -0.5581 & 0.406 \\
exact diag. (Refs. 11 and 34) & -0.5604 & 0.400 \\
$\infty \times \infty$ & & \\
our best WF & $-0.532(1)$ & 0.36 \\
spin-wave results (Ref. 12) & -0.540 & 0.25 \\
Capriotti et al. (Ref. 12) & -0.545 & 0.21 \\
\hline \hline
\end{tabular}

significantly lower energies than the simple $d^{+}$wave function, and has energies very close to the best ones available. More precisely, we find in the thermodynamic limit an energy per site of $e=-0.52 J$ for our variational wave function. Applying one Lanczos step leads to a small further improvement of the energy to $e=-0.53 \mathrm{~J}$. A summary of the energies and of the $120^{\circ}$ magnetization for the Heisenberg model are given in Table I and in Fig. 3.

Inspection of these results shows that our wave function has in the thermodynamic limit an energy only $0.013 \mathrm{~J}$ higher than the estimates of more sophisticated, but restricted to the undoped case, methods. ${ }^{12}$ Indeed, these latter methods use pure spin variational wave function as a starting point, restoring quantum fluctuations with a nonvariational method. Let us point out, however, that these methods are not giving an upper bound on the true ground-state energy, so the ground-state energy could in principle lie between this result and our variational one. Since our variational wave function already gives an excellent energy it would be interesting to check how the (nonvariational) methods used to improve the energy starting with a much cruder variational starting point would work with our variational wave function and which energy it would give. We leave this point for future investigation, however.

Our wave function shows a reduced but finite magnetic order that survives in the triangular Heisenberg antiferromagnet (THA). The $120^{\circ}$ magnetization of our wave function is reduced by the BCS pairing down to $72 \%$ of the classical value (see Fig. 7) which is somewhat larger than the spinwave result. Thus in addition to having an excellent energy, our wave function seems to capture the physics of the ground state of the Heisenberg system correctly. Let us note that the BCS order of the wave function is destroyed by the full Gutzwiller projector at half filling. So, despite the presence of a variational superconducting order parameter, the system is, of course, not superconducting at half filling. Somehow the BCS variational parameter helps to form singlets, which reduces the amplitude of the AF order. This is very similar to what happens for the $t-J$ model on the square lattice: the inclusion of a superconducting gap decreases the energy and decreases also the magnetization from $M \approx 0.9$ down to $M \approx 0.7$, which is somewhat larger than the best QMC estimates $(M \approx 0.6$, see Refs. 6 and 35$)$. Thus the wave-function mixing magnetism and a RVB gap seem to be interesting variationally, both in the square and triangular lattice, to restore spin fluctuations that were frozen in the pure classical magnetic wave function. The present work is an attempt to reproduce the magnetic order in the THA in terms of a fermionic representation, which gives results in good agreement with other methods. The great advantage of this approach is, of course, that the fermionic language allows to directly consider the case of hole and electron doping in the AF background, which is the case we consider in the following sections.

\section{B. Electron doping: $n \in[1,2]$}

Very few results exist away from half filling, so in order to have a point of comparison for our variational approach 


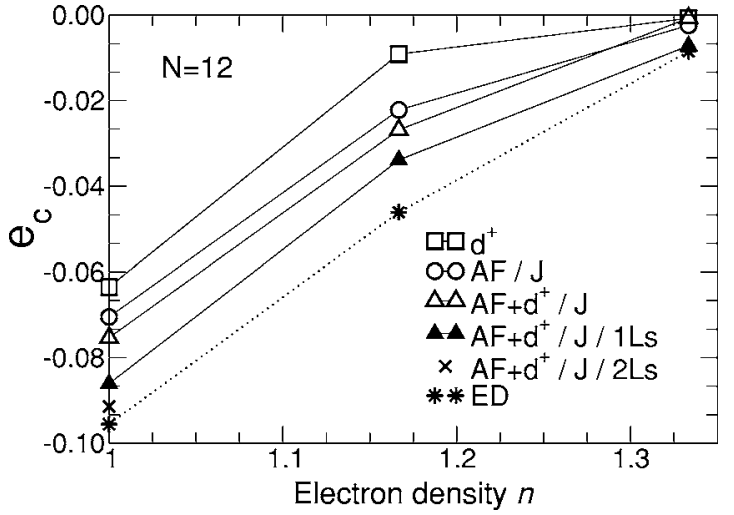

FIG. 4. Condensation energy per site versus the electron doping for a 12 site cluster for the different variational wave functions. We have done exact diagonalization (ED) for a 12 sites cluster with same periodic boundary conditions.

we will compare it with exact diagonalizations on very small clusters. Having ascertained that our wave function is indeed in good agreement with the exact results on a small cluster, we can then use it with confidence to describe much larger systems and extract the physics of the thermodynamic limit.

Therefore, we start by comparing on a 12 site cluster different wave functions with the exact-diagonalization results for the case of electron doping (see Fig. 4), since larger lattices are not readily available. Interestingly, it was found that even with only 2 Lanczos steps on our best wave function $\left(\mathrm{AF}+d^{+} / J\right)$ the energy has almost converged to the exact ground-state energy at half filling.

Note that small system size is the worst possible case for a VMC method since the simple variational wave function is not expected to reproduce the short-distance correlations well, as we fix the long-range magnetic correlations in our variational ansatz by imposing an on-site magnetic field, but we do not introduce short-range corrections. Variational Monte Carlo instead focus on the long distance properties, which will become dominant in the energy as the lattice size increases. Nevertheless, the short-range correlations contributes significantly to the energy on small lattices. One can thus expect on general grounds the energies to become increasingly good as the system size increases, provided that the correct long-range order has been implemented in the wave function. The Lanczos iterations allow one to correct this local structure of the wave function. Here we see that by changing this local structure our wave function is converging very fast to the ground state. This is a good indication that even away from half filling our wave function is quite efficient in capturing the physics of the system. Actually, the variance of the energy per site $\sigma^{2}$ reaches its maximum value for doping $x=\frac{1}{3}\left(\sigma^{2}=0.006\right)$, but applying one Lanczos step reduces drastically the variance $\sigma^{2}=0.0004$. At half filling, a variance-energy plot for the three functions $\mathrm{AF}+d^{+} / J / p \mathrm{Ls}$ $(p=0,1,2)$ allows one to extrapolate the energy at 0 variance, and we get an energy per site of $e=-0.61(1)$, which is very close to the exact result $e=-0.6103$.

Let us now use our wave function to describe large systems away from half filling. We now focus on a 108 site cluster, which is the largest cluster we can treat with a rea-

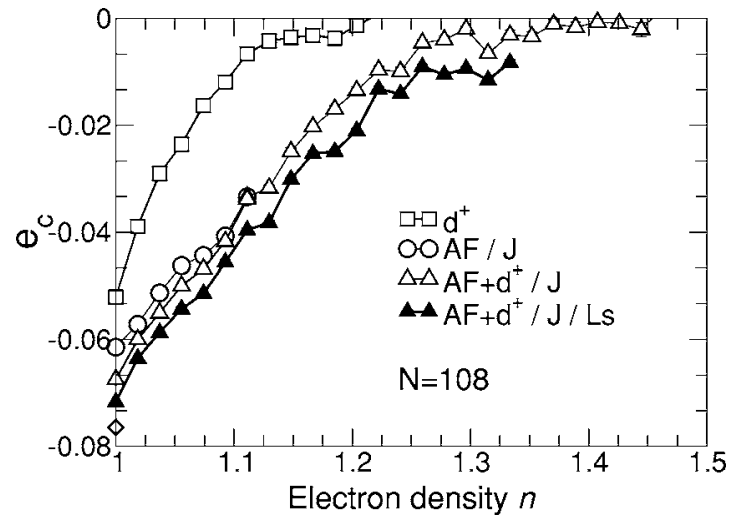

FIG. 5. Condensation energy per site $e_{c}$ versus electron doping for the 108 sites lattice. We show different wave functions and also the best estimate in the literature (Ref. 12 at half filling (open diamond).

sonable effort. We have first measured the condensation energy per site (see Fig. 5) for different types of instabilities. Very interestingly, the $\mathrm{AF} / J$ is even better than a simple $d_{x^{2}-y^{2}}+i d_{x y}$ RVB state. Moreover, the RVB order is only weakly increasing the condensation energy in presence of the antiferromagnetic background $\left(\mathrm{AF}+d^{+} / J\right)$. This is suggesting that superconductivity is only weakly present in the $t-J$ model when $n>1$ which is also confirmed by the measurement of the superconducting gap (see Fig. 6). The superconducting order of our best wave function is approximately 4 times smaller in amplitude and in range of stability than the $d$-wave pairing in a $10 \times 10$ square lattice with the same boundary conditions. For electron doping $\delta>0.04$ we find that the $d^{+}$BCS pairing symmetry has the same energies as the $d^{-}$one, and also as the wavefunctions with $d_{x^{2}-y^{2}}$ and $d_{x y}$ pairings.

Very strikingly, the $120^{\circ}$ magnetic order parameter is surviving up to high doping $\delta=0.4$, see Fig. 7. Long-range magnetic order at finite doping is potentially caused by a limitation of the VMC method, in that it is not possible in our calculation to model wave function with a finite correlation

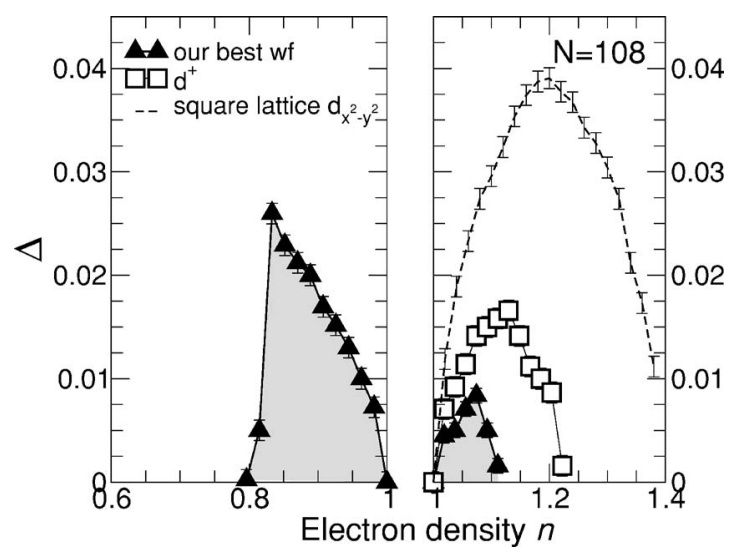

FIG. 6. Superconducting order parameter $\Delta$ for a 108 site triangular cluster in our best wave function (full triangles), in the $d^{+}$ wave function (open squares). For comparison we show the amplitude of the $d$-wave gap in a $10 \times 10$ square lattice (dashed line). 


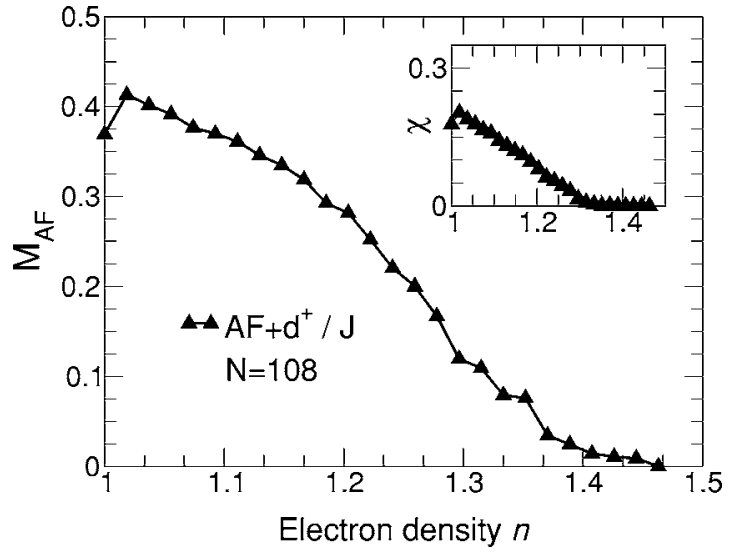

FIG. 7. Amplitude of the $120^{\circ}$ magnetic order $M_{\mathrm{AF}}$ measured in the $\mathrm{AF}+d^{+} / J$ for a 108 site cluster. Inset: the amplitude of the staggered spin-current $\chi$ in the same wave function.

length, i.e., short-range $120^{\circ}$ magnetic order. In our calculation, we can either totally suppress the long-range $120^{\circ}$ and get back to the Gutzwiller wave function, or use the longrange $120^{\circ}$ magnetic order that is highly stabilized by the potential energy. No intermediate scenario, such as incommensurate structures, is yet available in our calculations, but one can only expect the optimization of the magnetic structure to increase the region of stability for magnetism. We interpret this finding as an indication that the hole motion is not drastically modified by the presence of the noncollinear magnetic structure, so that short-range magnetic correlations will survive up to high electron doping. For the $t-J^{z}$ model on the square lattice, it is commonly understood that the Ising Néel order is not surviving high doping because of its costs in kinetic energy: whenever a hole wants to move in a antiferromagnetic spin background, it generates a ferromagnetic cloud. Therefore, good kinetic energies and Néel Ising order are not compatible. In our case, the three-sublattice order imposes no such constraint on the kinetic energy of the holes, because of the $120^{\circ}$ structure. We see this fact in our wave-function energies: the potential energy is improved when starting from the Gutzwiller wave function and adding $120^{\circ}$ correlations, but the kinetic energy is unchanged. Our best wave function has a better potential energy than the Gutzwiller wave function (and also than the different CFP wave function), but it also keeps the same kinetic energy. Therefore, this qualitatively explains why one can stabilize the three-sublattice magnetic phase for a large set of $J$ values. Finally, we note that the staggered spin-current pattern is also present for doping $\delta=[0,0.3]$ (see Fig. 7).

Interestingly, the Jastrow variational parameter $\alpha$ (8) is changing sign at $\delta=0.4$ when $M_{\mathrm{AF}} \approx 0$ : for $\delta<0.4(\delta>0.4)$ the Jastrow factor favors classical Ising (ferromagnetic) antiferromagnetic states. The VMC results show that the competition between the classical Ising configuration on the triangular lattice and the $120^{\circ}$ order is improving the energy. We argue that the classical Jastrow simulates with a good approximation quantum fluctuations around the $120^{\circ}$ order. Note that the Jastrow does not play the same role as the BCS pairing: the BCS pairing forms configurations of resonating singlets, and the Jastrow factor forms classical Ising configu-

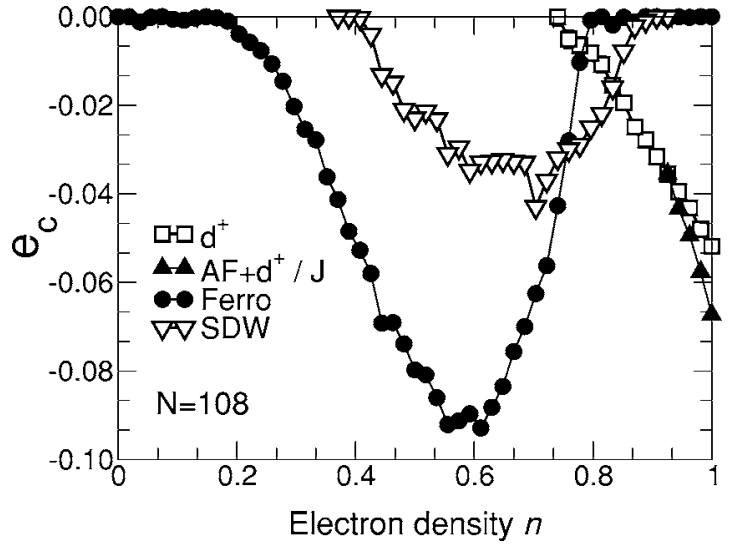

FIG. 8. Condensation energy per site $e_{c}$ for different wave functions in a 108 site cluster.

rations. It is also worth noting that at higher doping the Jastrow parameter is leading to a small condensation energy of $0.01 t$ for a large range of doping $(\delta=[0.4,0.8])$. It was checked that this gain in energy does not decrease with the size of the lattice and is also present for a square lattice geometry. We found also that for the small clusters (12 and 48 sites) the system was gaining a significant amount of energy when having a weak ferromagnetic polarization. Therefore, this is suggesting that the Gutzwiller wave function is not the best approximation of the ground state of the $t-J$ model in the high doping limit. Nevertheless, the Jastrow factor does not introduce long-range correlation and the variational wave function we introduce here is still a Fermi liquid.

Note that the variance of the energy per site $\sigma^{2}$ reaches its maximum value for doping $\delta=0.4$ for the $\mathrm{AF}+d^{+} / J$ with $\sigma^{2}=0.0008$, and applying one Lanczos step leads to $\sigma^{2}=0.0004$.

\section{Hole doping: $n \in[0,1]$}

For hole doping the scenario is strikingly different. The $120^{\circ}$ order is weakened in a strong $d^{+}$RVB background and disappears at doping $\delta=0.08$ (see Figs. 8 and 9). When superconductivity disappears, there is a first order transition to a commensurate spin density wave. No coexistence between superconductivity and the spin density wave was found. Then, a ferromagnetic phases emerges with a strong gain of condensation energy. Indeed the polarized states are leading to a strong gain in kinetic energy. This can be understood in the simple picture of the Stoner model, which gives a critical onsite repulsion related to the density of states: $U_{\mathrm{cr}}^{F}=1 / \rho\left(\epsilon_{F}\right)$. Ferromagnetism becomes favorable if $\epsilon_{F}$ is sitting at a sharp peak of $\rho(\epsilon)$. In the triangular lattice the tight binding density of states is strongly asymmetric and has a sharp peak at the $n=0.5$ electronic density lying at the Van Hove singularity. Note also that the simple $t-J$ model of a three site cluster with two electrons shows that in the $t>0$ the ground state is a singlet, whereas the ground state is a triplet in the $t<0$ case. This shows that the negative sign of $t$ with hole doping is inducing ferromagnetic correlations on a very small cluster. We find again trace of these correlations 


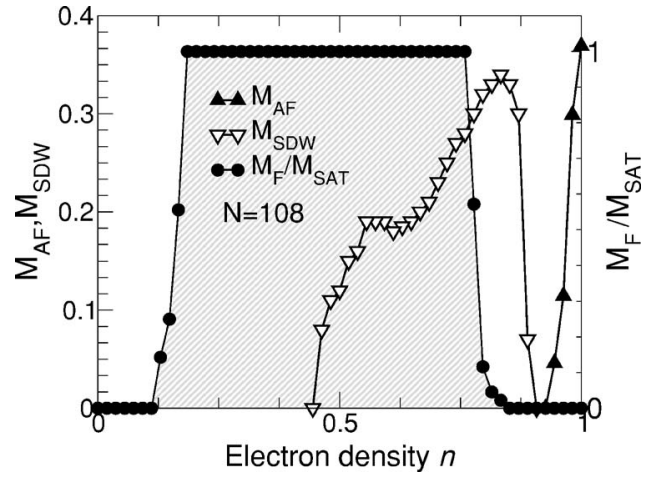

FIG. 9. Amplitude of the $120^{\circ}$ magnetic order measured in our best wavefunction for the 108 site cluster (left scale, full triangles) and the ratio of the polarization $M_{F}$ on the saturated polarization $M_{\text {sat }}$ in our best wave function (right scale, full circles). We show also the absolute magnetization $M_{\mathrm{SDW}}$ for the spin density wave wave function (left scale, see also Fig. 10).

and ferromagnetic tendencies in the range of electronic density $n \in[0.2,0.8]$ in our 108 site lattice. Such a ferromagnetic instability was also predicted in Ref. 21 by comparing the energy of the RVB wavefunction with an analytical calculation of the energy of the fully polarized state. We see that minimizing the energy by changing the variational onsite magnetic field leads to similar results.

Moreover, at $\delta=0.5$ doping, there is a nesting of the Fermi surface, with three possible $\mathbf{Q}$ vectors. Thus, it is reasonable to expect that a particle-hole instability of corresponding pitch vector $\mathbf{Q}$ is stabilized close to this doping. We have investigated the following instabilities: a commensurate charge density wave and a spin density wave. Interestingly, the commensurate spin density wave was stabilized. Indeed, we have found that the scattering between the $\mathbf{k}$ and $\mathbf{k}+\mathbf{Q}$ vectors introduced in the Hamiltonian $H_{\text {SDW }}$ (particle-hole channel) allow one to gain kinetic energy in the range of doping $\delta=[0.15,0.6]$. For sake of simplicity, we have only considered the mean-field Hamiltonian containing one of the three possible nesting vectors: $\mathbf{Q}_{N}=(\pi,-\pi / \sqrt{3})$. Finally, the phase is stabilized, when compared to the RVB and ferromagnetic phases, in the window $\delta=[0.16,0.24]$. Nonetheless, no coexistence between superconductivity and the spin density wave was found: the energy is minimized either for $\left[\Delta_{k}=0, f(Q) \neq 0\right]$ or $\left[\Delta_{k} \neq 0, f(Q)=0\right]$ depending on the doping, with $\Delta_{k}$ of $d_{x^{2}-y^{2}}+i d_{x y}$ symmetry type in the latter case. Measuring the on-site magnetization value, we found that the spin density wave is forming a collinear stripelike pattern in the spins degrees of freedom, whereas the charge is found to be uniformly distributed among the lattice sites, as expected (see Fig. 10). The amplitude of the on-site magnetization is shown in Fig. 9 as a function of doping.

\section{Phase diagram of the model}

Based on our wave function we can now give the phase diagram for the doped system on the triangular lattice. The phase diagram, summarizing the various instabilities discussed in the previous sections is show in Fig. 11. This phase

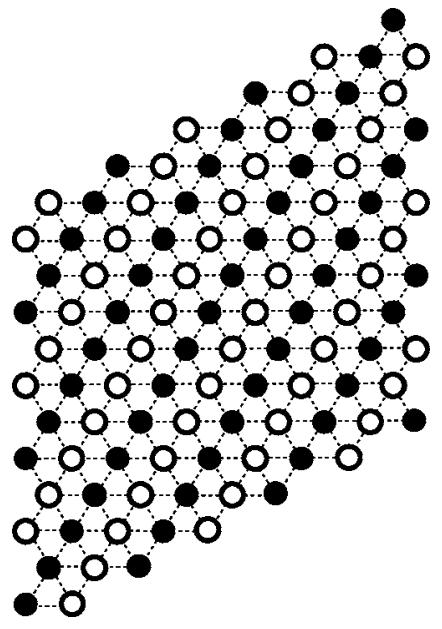

FIG. 10. On-site magnetization for each site of a 108 site lattice for the spin density wave function. Open (filled) circles denotes down (up) spins. The size of each circle is proportional to the respective amplitude of the on-site magnetization. We find that the spins forms a stripelike pattern, alternating ferromagnetic bonds in the $\mathbf{a}_{2}$ direction, and antiferromagnetic bond in the two other directions. The average on the lattice sites of the absolute value of the local magnetization is shown in Fig. 9.

diagram prompts for several comments. First one notices immediately that the competition between magnetism and superconductivity in this model depends crucially on the sign of the hopping integral (see Fig. 11).

For both hole and electron doping, the triangular lattice has a very different phase diagram from the square lattice one. ${ }^{38}$ In the square lattice, the AF order disappears at $\delta=0.1$ and the $d$-wave RVB dies at $\delta=0.4$ for the same value of $J$. In the triangular lattice, a similar stability of superconductivity exists on the hole side, but the electronically doped side is resolutely dominated by antiferromagnetic instabili-

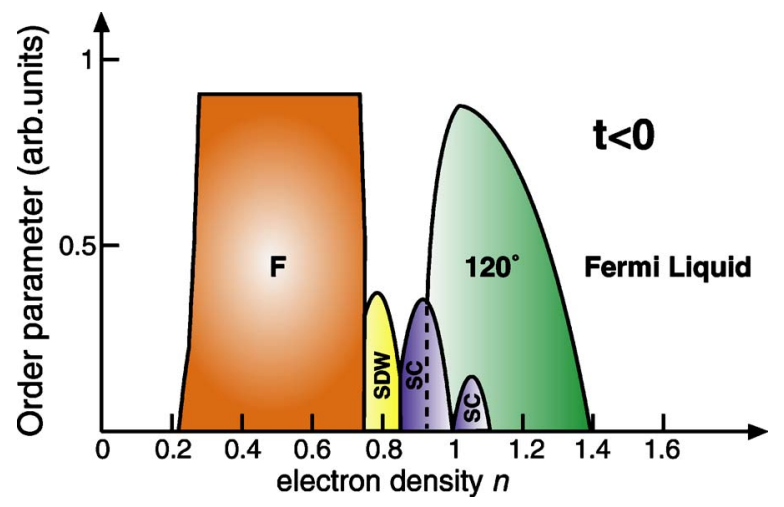

FIG. 11. (Color online) Cartoon picture of the phase diagram of the $t-J$ model we get with $t<0$. Here we sketch on an arbitrary scale the order parameter amplitude of the $120^{\circ}$ magnetic phase, the ferromagnetic phase $(F)$, the superconducting $d_{x^{2}-y^{2}}+i d_{x y}$ phase (SC), and the commensurate spin density wave (SDW). Note that for electron density $n>1.04$ and $n<0.96$, the energy is degenerated, within the error bars due to the Monte Carlo sampling, with the pairings $d_{x^{2}-y^{2}}, d_{x y}$ and $d_{x^{2}-y^{2}}-i d_{x y}$. The pitch vector of the commensurate spin density wave is $\mathbf{Q}_{N}=(\pi,-\pi / \sqrt{3})$, and this phase is depicted more in details in Fig. 10. 
ties. Our results, based on an improved class of wave functions, present marked differences with previous approximate results for the doped system. On the electron side, mean-field theories would have suggested that the long-range magnetic order state undergoes a first-order phase transition ${ }^{15}$ into a uniform $d^{+}$superconducting state at $\delta \approx 3 \%$ for values of $J$ similar to those considered here. A rationalization of these results would be that the frustration of the lattice, which was from the start the motivation of RVB as a competing state, disfavors magnetic order. Our results, where the Gutzwiller projection is treated exactly within the residual error bars due to the statistics, are in strong disagreement with this meanfield theory. Contrarily to the mean-field result, magnetism is dominant and the superconducting order is not favored on the electronic side. In addition, the $t-J$ model on the triangular lattice was expected to have a strong and large RVB instability, since the coordination number is higher than on most of the other lattices, and naively we would expect this to provide an easy way to form singlets. In this work we show that it is not the case: for electron doping the system is magnetic, and for hole doping the system is superconducting, but the range of superconductivity is not extraordinary large $(\delta<0.16)$, and smaller than on the square lattice.

Previous variational approaches ${ }^{21}$ were restricted to pure superconducting wave functions $d_{x^{2}-y^{2}}+i d_{x y}$ on a $t-t^{\prime}$ square lattice with $t=t^{\prime}$. In that work it was found that superconductivity is stabilized up to electron doping $\delta \approx 0.24$ and hole doping $\delta \approx 0.2$ for similar albeit slightly different values of $J / t(J / t=0.3)$. In our work, for the case of electron doping, which corresponds to the doping in the cobaltite experiments, our phase diagram, using the larger class of wave functions, is clearly completely different from this previous result, and the stabilization of the superconductivity in that case was clearly an artefact of the too restricted variational subspace. As show in Fig. 11 superconductivity is strongly weakened by the presence of three-sublattice magnetization and is present only in the range of electron doping $\delta=[0,0.12]$. On the contrary, for the case of hole doping, superconductivity had higher energy than ferromagnetic and spin density wave phases for $\delta>0.16$. We thus confirm that the previous results are not an artefact of their restricted variational subspace, and find an acceptable agreement for the phase diagram. However, we emphasize the presence of the spin density wave wave function that was not considered in the mentioned work and implies a small reduction of the superconductivity range.

Our calculation thus clearly prompts for a reexaminations of the arguments on the nature of superconductivity in a frustrated lattice. Clearly the noncollinear nature of the order parameter helps making the AF order much more stable to electron doping than initially anticipated. Understanding such issues is of course a very crucial and challenging question. Moreover, on the triangular lattice, no significant enhanced cooperative effect between magnetism and superconductivity seems to be observed: the electron doped side has a magnetic signature, and the hole doped side a superconducting one, but the two orders seem to exclude each other as much as they can, contrarily to what happens for the square lattice. Even in the parts of the phase diagram where coexistence is observed, coexistence between magnetism and su- perconductivity in the electron doped case shows again that superconductivity is decreased in the presence of strong long-range magnetic correlations.

\section{CONCLUSION}

In this paper we have presented a variational Monte Carlo study of the $t-J(J / t=0.4$ and $t<0)$ model on the triangular lattice, using extended wavefunction containing both superconductivity and noncollinear magnetism, as well as flux phase instabilities. The method we used to construct and sample the wave function is quite general and applicable to other lattices (honeycomb, kagome, ladders, etc.) as well as other symmetries (e.g., triplet superconductivity). It thus provides a general framework to tackle the competition between antiferromagnetism and superconductivity in frustrated systems. We obtained very good variational energies at half filling when comparing with other more sophisticated methods, specialized to the half-filled case. The fermionic representation of our wave function allows us to consider hole and electron doping. The most stable pairing corresponds to singlet pairing. We find that $d_{x^{2}-y^{2}}+i d_{x y}$ superconductivity is only weakly stabilized for electron doping in a very small window $(\delta=[0,0.12])$ and is much stronger and also appears in a wider range $(\delta=[0,0.16])$ in the case of hole doping. A commensurate spin density wave phase is leading to a gain in kinetic energy and is stabilized in the small window $\delta=[0.16,0.24]$ hole doping. Finally, ferromagnetism emerges in a wide range for hole doping $\delta=[0.24,0.8]$. Very surprisingly, the three-sublattice magnetism which is present at half filling extends to a very wide range of electron doping $\delta=[0,0.4]$ and is suppressed very fast in the case of hole doping $\delta=[0,0.08]$. The large extent of $120^{\circ}$ order for electron doping is responsible for the suppression of superconductivity. This feature was neither observed in previous VMC calculations, nor predicted by the mean field theories, and prompts for a reexamination of the question of the stability of magnetic order on a triangular system.

Our results show that, for electron doping, the square and triangular lattices behave in a very different way. For the square lattice, the $t-J$ Hamiltonian finds a domain of stability of superconductivity and a pairing symmetry that is very consistent with other methods. It is thus a natural candidate to investigate superconducting phases in systems such as the cuprates. For the case of the triangular lattice, the predicted phase diagram is dominated by antiferromagnetic instabilities, and superconductivity, albeit slightly present, is strongly suppressed. This clearly indicates that, contrarily to what was suggested by mean-field and previous variational calculations, the $t-J$ model itself is not a good starting point to tackle the superconductivity of the cobaltite compounds, where superconductivity is observed in the range of electron density $n=\left[1+\frac{1}{4}, 1+\frac{1}{3}\right]$. This model must be completed by additional ingredients to obtain a faithful description of the experimental system. Two missing ingredients in the simple $t-J$ model could solve this discrepancy and perhaps allow us to obtain a superconducting instability. On one hand, a strong Coulomb repulsion is expected in this type of compound. Such a long-range interaction is not taken into account in the 
$t-J$ model. Thus a Coulomb $V$ term should be added to get a $t-J-V$ model. On the other hand, in this paper, we have used a single-band model as a first step to study the Co-based oxides. However, it is quite possible that the multiband effect plays an essential role for superconductivity. ${ }^{36}$ The interaction between the three bands of the compound could play a nontrivial role in the physics of the $t-J$ model. Therefore, a study of the three-band model could also be of interest. Such an analysis can be done by extending the methods exposed in this paper to these more complicated models.

\section{ACKNOWLEDGMENTS}

We are grateful to Federico Becca and Antoine Georges for useful discussions. This work was supported by the Swiss National Fund and by NCCR MaNEP.
${ }^{1}$ P. W. Anderson, Science 235, 1196 (1987).

${ }^{2}$ H. Yokoyama and H. Shiba, J. Phys. Soc. Jpn. 57, 2482 (1988).

${ }^{3}$ C. Gros, Phys. Rev. B 38, 931 (1989).

${ }^{4}$ G. Kotliar, Phys. Rev. B 37, 3664 (1988).

${ }^{5} \mathrm{~T}$. Giamarchi and C. Lhuillier, in Condensed Matter Theories, edited by V. Aguilera-Navarro (Plenum, New York, 1989), Vol. 5.

${ }^{6}$ T. Giamarchi and C. Lhuillier, Phys. Rev. B 43, 12943 (1991).

${ }^{7}$ C. T. Shih, Y. C. Chen, C. P. Chou, and T. K. Lee, Phys. Rev. B 70, 220502 (2004).

${ }^{8}$ A. Paramekanti, M. Randeria, and N. Trivedi, Phys. Rev. B 70, 054504 (2004).

${ }^{9}$ P. W. Anderson, P. A. Lee, M. Randeria, T. M. Rice, N. Trivedi, and F. C. Zhang, J. Phys.: Condens. Matter 16, R755 (2004).

${ }^{10}$ D. Huse and V. Elser, Phys. Rev. Lett. 60, 2531 (1988).

${ }^{11}$ B. Bernu, C. Lhuillier, and L. Pierre, Phys. Rev. Lett. 69, 2590 (1992).

${ }^{12}$ L. Capriotti, A. E. Trumper, and S. Sorella, Phys. Rev. Lett. 82, 3899 (1999).

${ }^{13}$ G. Baskaran, Phys. Rev. Lett. 91, 097003 (2003).

${ }^{14}$ B. Kumar and B. S. Shastry, Phys. Rev. B 68, 104508 (2003).

${ }^{15}$ Q.-H. Wang, D.-H. Lee, and P. A. Lee, Phys. Rev. B 69, 092504 (2004).

${ }^{16}$ K. Takada, H. Sakurai, E. Takayama-Muromachi, F. Izumi, R. A. Dilanian, and T. Sasaki, Nature (London) 53, 422 (2003).

${ }^{17}$ R. E. Schaak, T. Klimczuk, M. Foo, and R. J. Cava, Nature (London) 424, 527 (2003).

${ }^{18}$ D. J. Singh, Phys. Rev. B 61, 13397 (2000).

${ }^{19}$ P. W. Anderson, Mater. Res. Bull. 8, 153 (1973).

${ }^{20}$ P. Fazekas and P. W. Anderson, Philos. Mag. 30, 423 (1974).
${ }^{21}$ T. Watanabe, H. Yokoyama, Y. Tanaka, J. Inoue, and M. Ogata, J. Phys. Soc. Jpn. 73, 3404 (2004).

${ }^{22}$ J. P. Bouchaud, A. Georges, and C. Lhuillier, J. Phys. (Paris) 49, 553 (1988).

${ }^{23}$ D. Ceperley, G. V. Chester, and K. H. Kalos, Phys. Rev. B 16, 3081 (1977).

${ }^{24}$ R. Jastrow, Phys. Rev. 98, 1479 (1955).

${ }^{25}$ G. H. Wannier, Phys. Rev. 79, 357 (1950).

${ }^{26}$ E. Heeb and T. M. Rice, Europhys. Lett. 27, 673 (1993).

${ }^{27}$ E. Heeb, Ph.D. thesis, ETHZ (Zürich, Switzerland), 1994.

${ }^{28}$ F. Becca, Ph.D. thesis, SISSA (Trieste, Italy), 2001.

${ }^{29}$ S. Sorella, Phys. Rev. B 64, 024512 (2001).

${ }^{30}$ C. J. Umrigar, K. G. Wilson, and J. W. Wilkins, Phys. Rev. Lett. 60, 1719 (1988).

${ }^{31}$ D. S. Rokhsar, Phys. Rev. Lett. 65, 1506 (1990).

${ }^{32}$ C. Honerkamp, Phys. Rev. B 68, 104510 (2003).

${ }^{33}$ P. Sindzingre, P. Lecheminant, and C. Lhuillier, Phys. Rev. B 50, 3108 (1994).

${ }^{34}$ B. Bernu, P. Lecheminant, C. Lhuillier, and L. Pierre, Phys. Rev. B 50, 10048 (1994).

${ }^{35}$ A. W. Sandvik, Phys. Rev. B 56, 11678 (1997).

${ }^{36}$ W. Koshibae and S. Maekawa, Phys. Rev. Lett. 91, 257003 (2003).

${ }^{37} H_{\mathrm{MF}}$ is gauge invariant, however, the gauge plays a nontrivial role in the projected wave function. Indeed, the kinetic energy of the projected wave-function depends on the chosen gauge. One cannot exclude that another choice of the gauge could lead to a better wave function.

${ }^{38}$ In the square lattice the sign of $t$ plays no role because of the particle-hole symmetry of a bipartite lattice. 